\title{
High resolution electron microscopy of alloys
}

\author{
G K DEY* and S BANERJEE \\ Materials Science Division, Bhabha Atomic Research Centre, Mumbai 400 085, India
}

\begin{abstract}
High resolution electron microscopy (HREM) has emerged as a very powerful tool for probing the structure of metals and alloys. It has not only helped in unravelling the structure of materials which have been at the forefront of novel materials development such as quasicrystalline phases and high temperature superconducting compounds, but also is fast becoming a technique for solving some outstanding issues in the case of the commercial alloys thereby helping alloy development. In addition to the determination of the structures of phases, this tool is used for obtaining a first hand information of the arrangement of atoms around the various types of crystallographic defects and interphase interfaces. This mode of microscopy allows direct observation of orientation relationships between two phases across interfaces. HREM can be used for the direct examination of the prenucleation process. Initial stages of nucleation can also be studied readily in amorphous alloys, precipitation hardening alloys like maraging steels and in those systems where the formation of the omega phase occurs.

This presentation describes some results of HREM studies on various alloys, commercial as well as alloys of scientific interest, where some of the aforementioned aspects have been examined. The specific examples cited pertain to metallic glasses, NiTi shape memory alloys, $\mathrm{Ni}-\mathrm{Mo}, \mathrm{Zr}-\mathrm{Nb}$ and $\mathrm{Ti}-\mathrm{Al}$ alloys.
\end{abstract}

Keywords. High resolution electron microscopy (HREM); phase; interface.

\section{Introduction}

It has been a long-standing desire of scientists to see atomic arrangements in solids directly, for such direct observations can resolve many issues concerning the structures of the constituent phases. The ability to see the atomic arrangement also permits observation of deviations from the ideal structure, thereby providing a new insight into the world of crystal defects. With the advent of high resolution electron microscopy (HREM), the dream of seeing the arrangement of atoms in solids has been realized. This mode of microscopy is of interest for resolving the structures of unknown phases, for studying various types of crystal defects and for ascertaining the arrangement of atoms at various interfaces (Spence 1988). This type of microscopy differs from the conventional form of transmission electron microscopy (TEM) in that it makes use of phase contrast in image formation (Van Landuyt et al 1991). The images are formed by using two or more beams, which are allowed to interface. In conventional TEM, diffraction contrast is made use of and the image is made from either the transmitted beam or one of the diffracted beams. The resolution of the electron microscope $\left(r_{\text {opt }}\right)$ depends not only on the wavelength of the electron beam $(\lambda)$, but also is governed by the aberration of the image forming lenses present in the electron microscope. The equation relating these quantities with the resolution of

\footnotetext{
*Author for correspondence
}

the microscope can be expressed as (Goodhew and Humphreys 1987)

$$
r_{\mathrm{opt}}=1 \cdot 21 \lambda^{3 / 4} C_{\mathrm{s}}^{1 / 4}
$$

In modern day microscopes, great emphasis is laid on minimizing the spherical aberration coefficient $\left(C_{s}\right)$ of the objective lens so as to improve the resolution of the instrument as much as possible. The resolution of the modern HREM is under $0.2 \mathrm{~nm}$ (point to point) even with a fair amount of specimen tilt. These microscopes are, therefore, capable of resolving the structures of different phases in most metals and alloys. A further improvement in the resolution has been brought about by the introduction of microscopes equipped with the field emission gun. The field emission gun reduces the instrumental contribution to the chromatic aberration; as a result of this information from planes of lower interplanar spacings are also transferred to the image (Williams and Carter 1996).

HREM has been used for the resolution of the structures of the phases present in a variety of materials. In recent years the structures that have been very successfully probed by this form of microscopy include those of quasicrystalline phases and of high temperature oxide superconductors (Shindo et al 1988; Williams and Carter 1996). The use of this form of microscopy in chemical and physical sciences has led to the delineation of the structures of various chemicals and minerals. HREM has emerged as a very powerful tool for probing the 
structure of the electronic materials. The multilayered structures of contemporaneous interest for their desirable properties from scientific as well technological viewpoints are being examined in considerable detail using this form of microscopy. HREM has been particularly useful in this area because of its ability to resolve the interface structures in these materials. This form of microscopy is also being extensively used in the study of the nanocrystalline phases.

It is possible to monitor several phase transformations on the atomic scale by carrying out in situ phase transformation studies in HREM. The aforementioned discussions indicate that this form of microscopy has very important applications not only in all areas of materials science but also in diverse areas such as biology and mineralogy. This paper aims to provide a brief account of the use of this technique in the study of some aspects of alloy phases. Unlike in conventional microscopy, in this form of microscopy the images have to be often processed and simulated images generated for comparison with experimentally observed images for a complete understanding of the structure (Williams and Carter 1996). Therefore, before coming to the use of this tool a brief description of these two important aspects related to this form of microscopy is presented.

\subsection{Image simulation}

Image simulation is necessary to ascertain the structures of phases and of the defects in any material. This is necessitated by the fact that the observed image has only the intensity information and not the phase information. In order to interpret the image, it is necessary to assume a structure of the material, simulate the image and then by comparison with the observed image, find out how closely the simulated image approaches the observed image. If there are differences between the two then the assumed structure is modified and the simulation process is repeated. If comparison reveals that the two are almost identical then it is very likely that the assumed structure replicates the actual structure of the material. Though this methodology may appear to be quite simple, it is not so in reality because the image characteristics are sensitive to several factors. The most important ones among these are: (i) the thickness of the specimen, (ii) the defocus of the objective lens, and (iii) the precise alignment of the beam with respect to both the specimen and the optic axis. It is possible to obtain the same simulated image using two different structures and one has to exercise caution while drawing inferences about the structure of a phase by having recourse to image simulation. There are four different ways of image simulation: (i) the reciprocal space formalism; (ii) the fast Fourier transform (FFT) formalism; (iii) the real space approach; and (iv) the Bloch-wave approach. A detailed account of these different techniques of image simulation has been given by Williams and Carter (1996).

\subsection{Image processing}

Unlike images obtained under diffraction contrast, the images obtained by phase contrast are subjected to a considerable amount of image processing. The reason for this is as follows. There is a need to make the images sharp because of the fact that HREM images are often made of dark and bright dots and these represent atomic positions. In order to understand the structure, it is necessary to make a clear distinction between the dots and the interveining regions in order to extract meaningful information about the structure. Image processing is primarily carried out for two reasons. First because of the need to make the images look sharp, more even in contrast or to increase the contrast. Secondly, there may be a need to quantify the information contained in the image. Though attempts have been made to process images or modify images by choosing different grades of printing paper, developers, and emulsions, modern image processing requires processing by using a computer. The three important requirements in this context are: (i) to create a digital image, (ii) to use the appropriate software for processing the image, and (iii) to use a computer to process the data in a reasonable period of time with sufficient accuracy.

While processing the image it is important to consider the changes brought about by the processing technique. Only after keeping this fact in mind it is possible to compare the actual image with the processed image in a meaningful manner.

In this paper the application of HREM in the following three areas of alloy phases has been dealt with: (i) structures of novel or unknown phases, (ii) initial stages of precipitation in alloys, and (iii) structures of the various types of interfaces in alloys.

\section{Studies on the structures of novel phases}

HREM has been extensively used for studying the structure of novel phases. The study of quasicrystalline phases is one of the several instances where HREM has been successfully used for obtaining unequivocal evidence about the structure of the material (Hirabayashi and Hiraga 1987; Nissen and Beeli 1991). The structures of quasicrystalline phases have been an enigma for quite some time. Diffraction techniques were not successful in unravelling these structures fully and the five-fold rotational symmetry observed in the diffraction patterns was difficult to visualize in the real space (Hirabayashi and Hiraga 1987; Nissen and Beeli 1991). The idea that quasicrystallinity existed in some materials faced considerable opposition from those who preferred to describe 
the structure in such materials in terms of multiple twinning of the perfectly crystalline lattice. HREM observation showed for the first time that these phases indeed have forbidden rotational symmetries in atomic arrangement. The study of quasicrystals by HREM continues to be very important because these materials lack the translational periodicity present in crystalline materials. Though these lack translational periodicity, these are highly ordered (Williams and Carter 1996). This is evident from the high resolution micrograph shown in figure 1 obtained from an $\mathrm{Al}$ based quasicrystal. This micrograph clearly demonstrates the kind of order present in these materials. Selected area diffraction (SAD) patterns from these materials show distinct spots like in those obtained from crystalline materials. However, these spots do not correspond to sets of planes as they do in the case of crystalline materials. The dots in HREM images of these materials are aligned along specific directions as in the case of crystalline materials and it has been shown that at least for decagonal quasicrystals, each dot indeed corresponds to a column of atoms (Williams and Carter 1996). It is not necessary to have translational periodicity along the column for each dot to represent one column. This indicates that for HREM, the atoms are needed to be aligned in columns because this is a projection technique, but the distribution along the column is not so important. The study of quasicrystals also indicates that SAD and HREM should be used in a complementary manner.

Another fascinating example of the use of HREM in the determination of the structure of the alloy phases is the investigation of the formation of an Al-rich poly typic phase in some of the intercellular regions in laser processed specimens of Ni coated with Mo and Al (Dey et al 1994). Occurrence of such phases is extremely rare

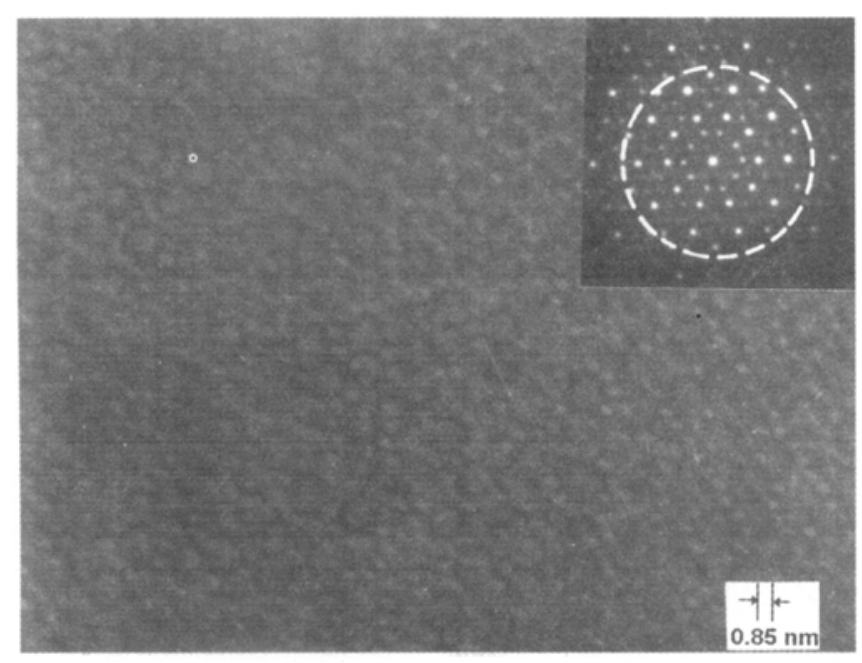

Figure 1. HREM image of a Al-14 Mn quasicrystalline alloy along the five-fold direction. in metallic systems. This phase has been found to be akin to the series of modulated structures based on the $\mathrm{Al}_{5} \mathrm{Mo}$ intermetallic phase (hexagonal; $a=0.489 \mathrm{~nm}$ and $c=0.880 \mathrm{~nm}$ ). The long period (modulated) derivatives of this structure comprise periodic arrangements of nonconservative faults, which correspond to insertion, or removal of Al-layers. HREM images of the modulated phase, exhibited periodically spaced lattice fringes (Dey et al 1994). Hairpin arrangements leading to merger or dissociation of faults could also be observed in some regions of this phase. The measured spacing of $1.7 \mathrm{~nm}$ between the successive faults was in agreement, to a first approximation, with the nine-layer periodicity proposed for this structure by Dey et al (1994).

There are numerous other examples where the HREM technique has been used for studying the structure of new phases (Spence 1988). The understanding of the structure of high temperature superconductors has improved considerably due to this technique (Shindo et al 1988). Imaging of the structure of these compounds along with extensive image simulation has been carried out in order to affix the positions of the oxygen atoms in the lattice of these technologically important materials (Shindo et al 1988).

\section{Studies on initial stages of precipitation in alloys}

In many phase transformations there are pretransformation effects, which are manifested in the electron diffraction patterns obtained from the parent phase. These can assume various forms such as satellite reflections, streaks and other forms of rel rods (Chandrasekhar 1993). In metallic systems, two important transformations where these effects are very conspicuous are the omega transformation and the martensitic transformation. A direct observation of the lattice of the parent phase where these pretransformation effects are occurring yields vital clues as to the mechanism by which such transformations initiate. Efforts have also been made to observe the very initial stages of product phase formation in transformations where pretransformation effects are not so prominent. It is very difficult to observe these by conventional microscopy because of the very small size of the nuclei. However, HREM is a very powerful tool for studying the initial stage of the transformation (i.e. nucleation stage).

The initial stages of omega formation have been studied in many alloys by using HREM (Tewari et al 1998; Schryvers and Tanner 1990). In these studies, attempts have been made to see the changes in the positions of the planes of the parent $\beta$ phase where the transformation has just set in. These observations have been made in $\mathrm{Zr}$ based omega forming systems as well as in $\mathrm{Ti}$ based alloy systems (Tewari et al 1998; Schryvers and Tanner 1990). It has been successfully shown by HREM that 
the collapse of the planes indeed takes place on the $\{111\}_{\beta}$ planes.

The initial stages of precipitation have been studied in a variety of alloys. One class of alloys where this can be very effectively studied comprises amorphous alloys (Savalia et al 1996). This is because of the fact that parent phase is amorphous and the lattice fringes corresponding to the crystalline nuclei against such a background become very easily visible. The periodic arrangement of atoms is absent in the amorphous phase and hence the image contrast arising from it in conventional microscopy is devoid of any notable features. In phase contrast, however, the amorphous structure exhibits some very characteristic features. Often the amorphous phase has very small regions, which are crystalline. These can be of the size of the crystalline nuclei or can be larger. In either case these influence the properties of the amorphous phase significantly. These crystalline regions also affect the thermal stability of amorphous alloys as these act as nucleation sites for the nucleation of new crystalline phases. High resolution electron microscopy is the right tool for studying the nature, size and identity of such small regions. It is, therefore, possible not only to ascertain the size and shape of the nuclei but also the nature of the phases emerging in the amorphous matrix. This mode of microscopy can detect the presence of very small crystalline regions, thus confirming the fully amorphous or partially crystalline nature of the specimen. The example cited here pertains to the initial stages of nucleation in a $\mathrm{Zr}$ based metallic glass. Electron diffraction showed one intense diffuse halo followed by another halo of lesser intensity-a feature that is characteristic of the amorphous phase. A high resolution electron micrograph obtained from such a specimen is shown in figure 2 . It can be seen in this micrograph that lattice fringes are present in several small regions, which are sparsely distributed in the amorphous matrix. The lattice planes in the fringed regions were found to be separated by about $0.25 \mathrm{~nm}$, a spacing closely matching with $d_{110}$ of $\beta-\mathrm{Zr}$ (bcc, $a=0.358 \mathrm{~nm}$ ) (Savalia et al 1996). The average size of such small crystalline regions was found to be about $3.0 \mathrm{~nm}$. The presence of these small crystalline domains in the as-quenched structure of this metallic glass could be established only by HREM; the material appeared to be fully amorphous in XRD, selected area diffraction (SAD) and conventional TEM. These quenched-in crystalline nuclei play a major role in the process of crystallization and determine the thermal stability of the amorphous phase. Often the crystallization of metallic glass involves the growth of such quenched-in crystalline nuclei (athermal nucleation) (Savalia et al 1996). Crystallization at temperatures where isothermal nucleation alone is occurring in the amorphous phase and the nucleation rate is very rapid, leads to the formation of very fine crystals which can be best described as nanocrystalline phases. These are very small crystallites, which have impinged before any substantial growth due to

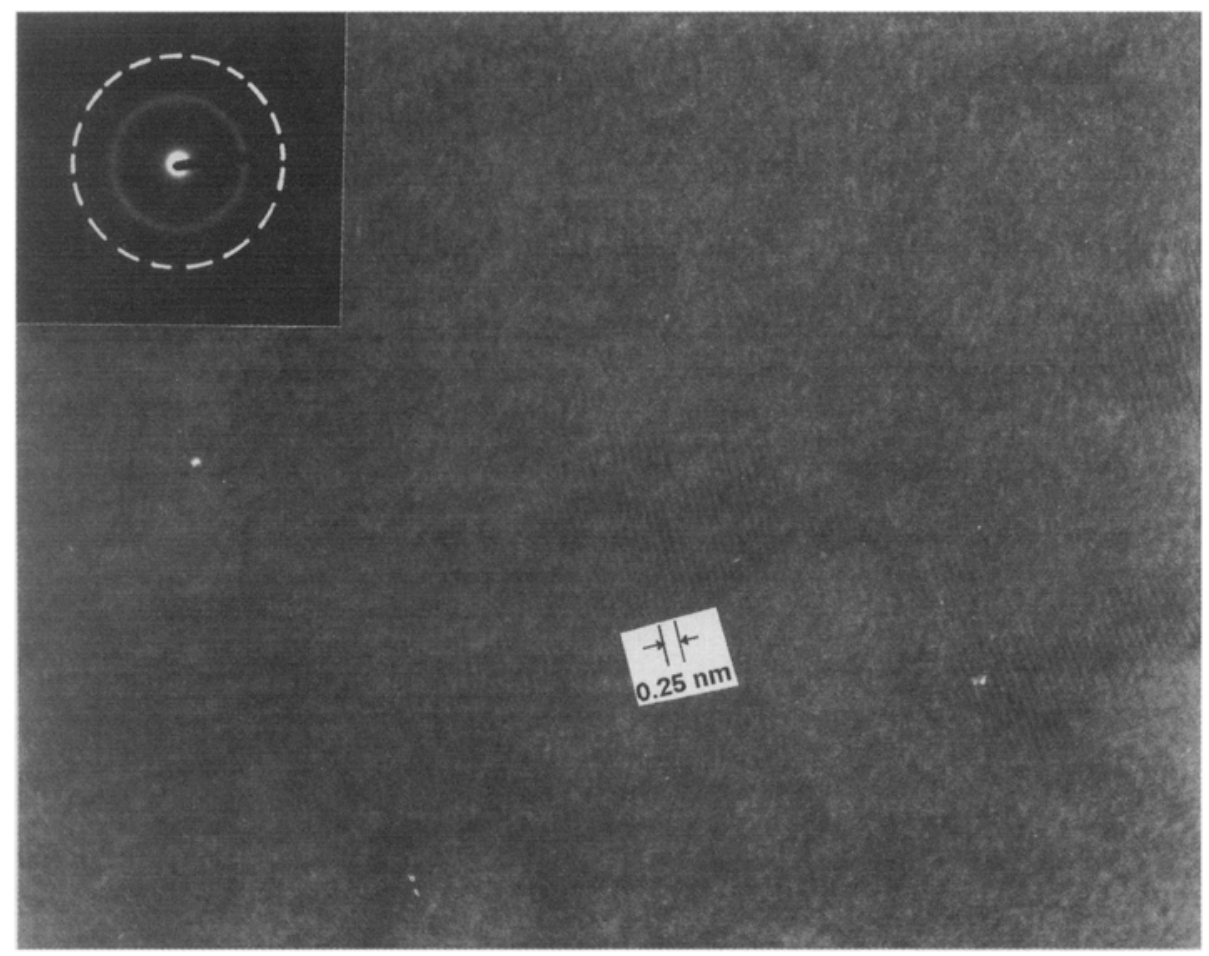

Figure 2. HREM picture showing the presence of a crystalline region in the amorphous phase. 
profuse nucleation. The formation of a nanocrystalline phase during the crystallization of a metallic glass has been studied by Dey et al (1998) using HREM.

\section{Studies on the structures of various types of interfaces in alloys}

For studying these interfaces near atomic resolution is required. Since such a resolution is readily available with a HREM, this mode of microscopy has been extensively used for the study of interfaces (Williams and Carter 1996). It has been shown that the study of the interfaces between grains become easier if a low index plane in at least one and preferably both the grains is parallel to the interface (Williams and Carter 1996). Only tilt boundaries have been studied by HREM. It is also possible to see the presence of amorphous layers at the interface. In addition, the interface between two different phases can be seen to find out the presence of ledges or steps and the relative orientation of the lattices. In this paper studies on interfaces in the following systems are presented.

\subsection{Ni-Mo alloys}

The ordering processes occurring in these alloys have been examined extensively both by conventional microscopy as well as by HREM (Banerjee et al 1988; Kulkarni
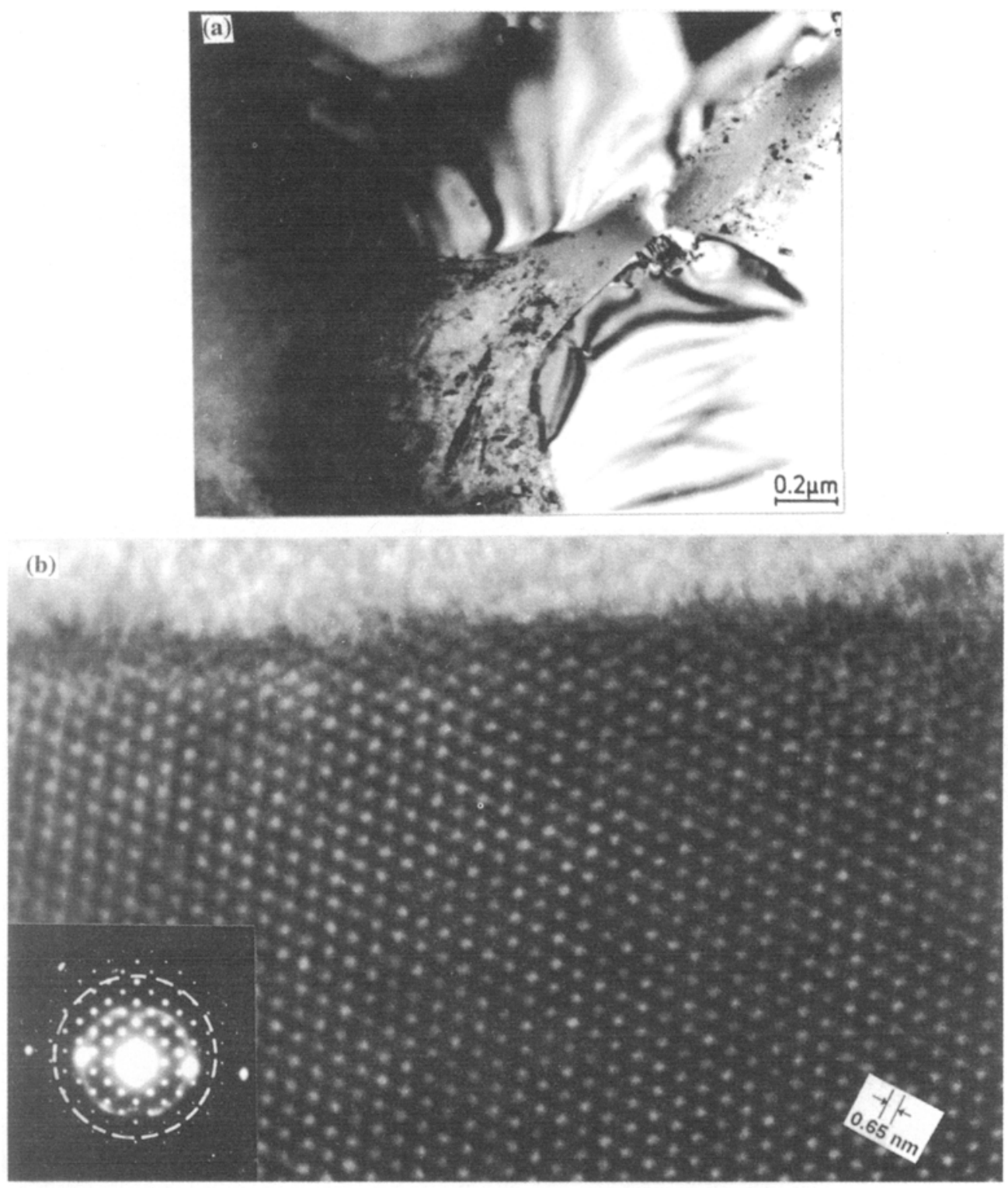

Figure 3. (a) Bright field micrograph showing a particle of the $\mathrm{Ti}_{2} \mathrm{Ni}$ phase in micropyretically synthesized $\mathrm{NiTi}$. (b) HREM picture showing the $\mathrm{Ti}_{2} \mathrm{Ni}$ particle and the interface with the matrix phase 
and Banerjee 1988). HREM has also been used to study the nature of the intermetallic precipitates and the various types of defects occurring in these precipitates. The sigma phase is one such precipitate in these alloys. The sigma phase has been found to have various types of interfaces. The important one among these is due to the formation of the few atomic layers of a second phase having an orthorhombic structure (Kulkarni and Banerjee 1988).

\subsection{NiTi alloys}

These alloys are of interest because these show shape memory (Banerjee and Madangopal 1996). Because of this reason these alloys find application at several places. The study of the microstructure of $\mathrm{NiTi}$ at an atomic level is very important because it allows a better understanding of the shape memory effect. The microstructure of the equiatomic NiTi alloy comprises of the B19 martensitic phase and the parent B2 phase. The martensitic phase is internally twinned whereas the parent B2 phase can have antiphase domain boundaries in it. In NiTi, various types of twins have been seen. These can be broadly categorized as $\{110\}$ and $\{111\}$ twins. The boundary structure of the various types of twins in the B19 martensitic structure of the shape alloy has been observed in the edge on condition. It has been seen that

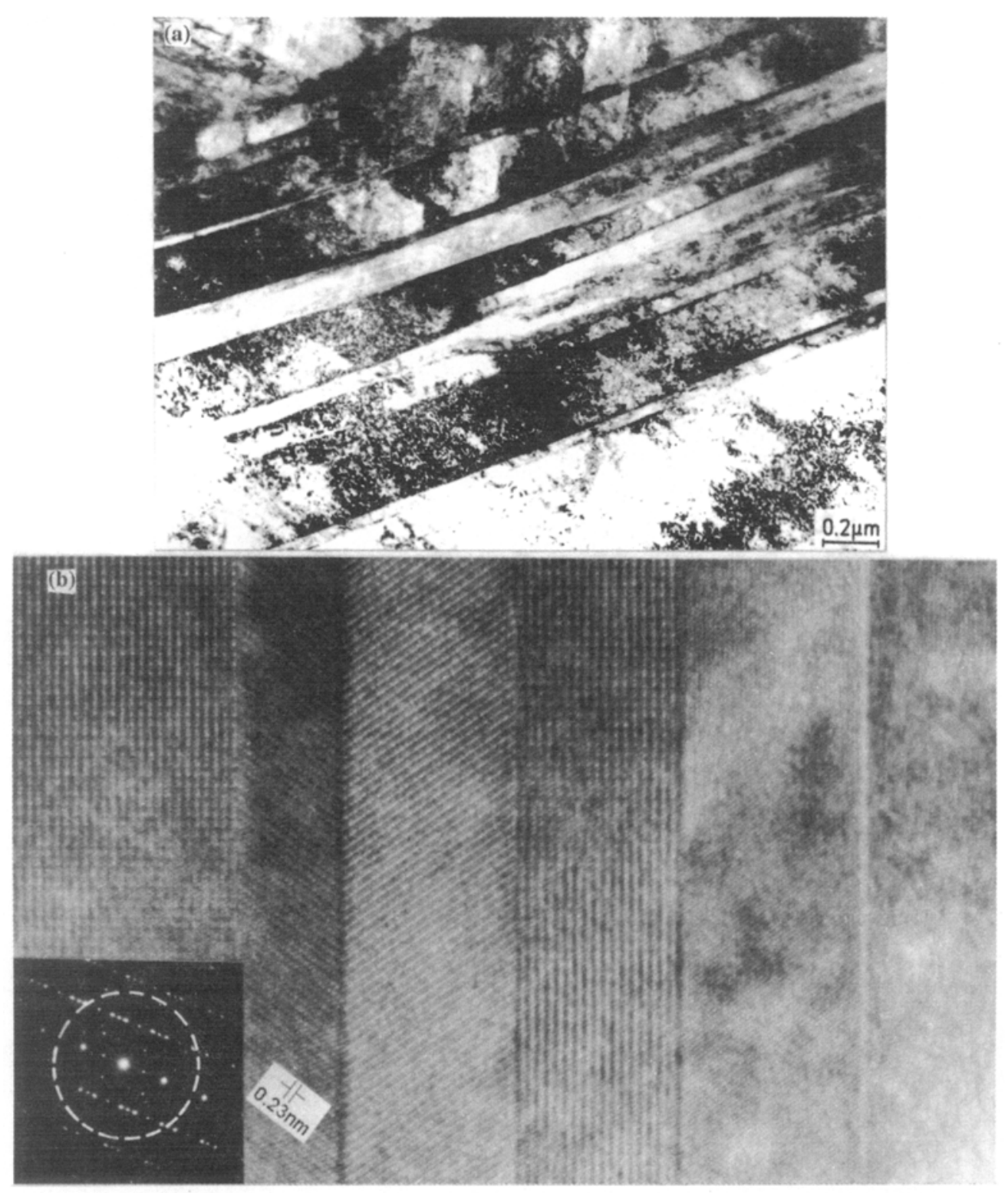

Figure 4. (a) Bright field micrograph showing the microstructure of micropyretically synthesized TiAl. (b) HREM image showing the microstructure of micropyretically synthesized TiAl alloy. The $\alpha_{2} / \gamma$ and the $\gamma / \gamma$ interfaces can be clearly seen in this micrograph. The $\gamma / \gamma$ interface is a twin interface. 
the type I and the compound twins exhibit the well-defined crystallographic features of these boundaries. One of the topics of controversy has been regarding the existence of the ledge or the step structures at the irrational boundaries. Knowles (1982) has shown the existence of such features at the boundaries whereas Nishida et al (1995) have shown that neither ledges nor steps are present at the interface of these boundaries. It has been seen in this alloy that these twins are very narrow in width and for most of the twins, the interfaces look very straight. However, when high resolution imaging of the interface has been carried out it has been observed that the interface looks very different in the sense that the some of the interfaces appear to be narrowing down to make a cone. A detailed account of HREM investigations on this alloy will be presented elsewhere (Dey 1998). In addition to the $B 2$ and the martensitic phases, it is also possible to see the presence of a precipitate phase in this alloy which is $\mathrm{Ti}_{2} \mathrm{Ni}$ phase (figure $3 \mathrm{a}$ ). This phase has a complex cubic structure with a lattice parameter of $a=1.1278 \mathrm{~nm}$. A lattice resolution micrograph obtained from this phase is shown in figure $3 \mathrm{~b}$. The interface between this phase and the matrix phase was not a coherent interface.

\subsection{TiAl alloys}

The TiAl ( $\gamma$ phase) based alloys have acquired considerable significance in recent times because of their technologically attractive properties (Kim 1992). Continuous endeavor is being made to develop TiAl alloys with superior properties, a task in which a full understanding of the microstructure of the alloy is of utmost importance. HREM has been used to resolve many of the outstanding issues pertaining to the microstructure of these alloys. The $\gamma-\gamma$ interface and $\gamma-\alpha_{2}$ interfaces have been examined in considerable detail (Singh and Howe 1992). The nature of the various mixed boundaries in the $\gamma$ phase of the lamellar structure in this type of compound has also been examined by HREM (Yang and Wu 1992). Particular attention has been given to the atomic structure of twin boundaries (TB), pseudo twin boundaries (PTB), order domain boundaries (ODB) and antiphase domain boundaries (Singh and Howe 1992). The mechanism of formation of the various types of boundaries has also been examined in considerable detail (Yang et al 1993). It has been shown that the mixed twin boundaries (MTB) exist in the $\gamma$ phase of the lamellar structure (Yang et al 1992). The mixed boundary

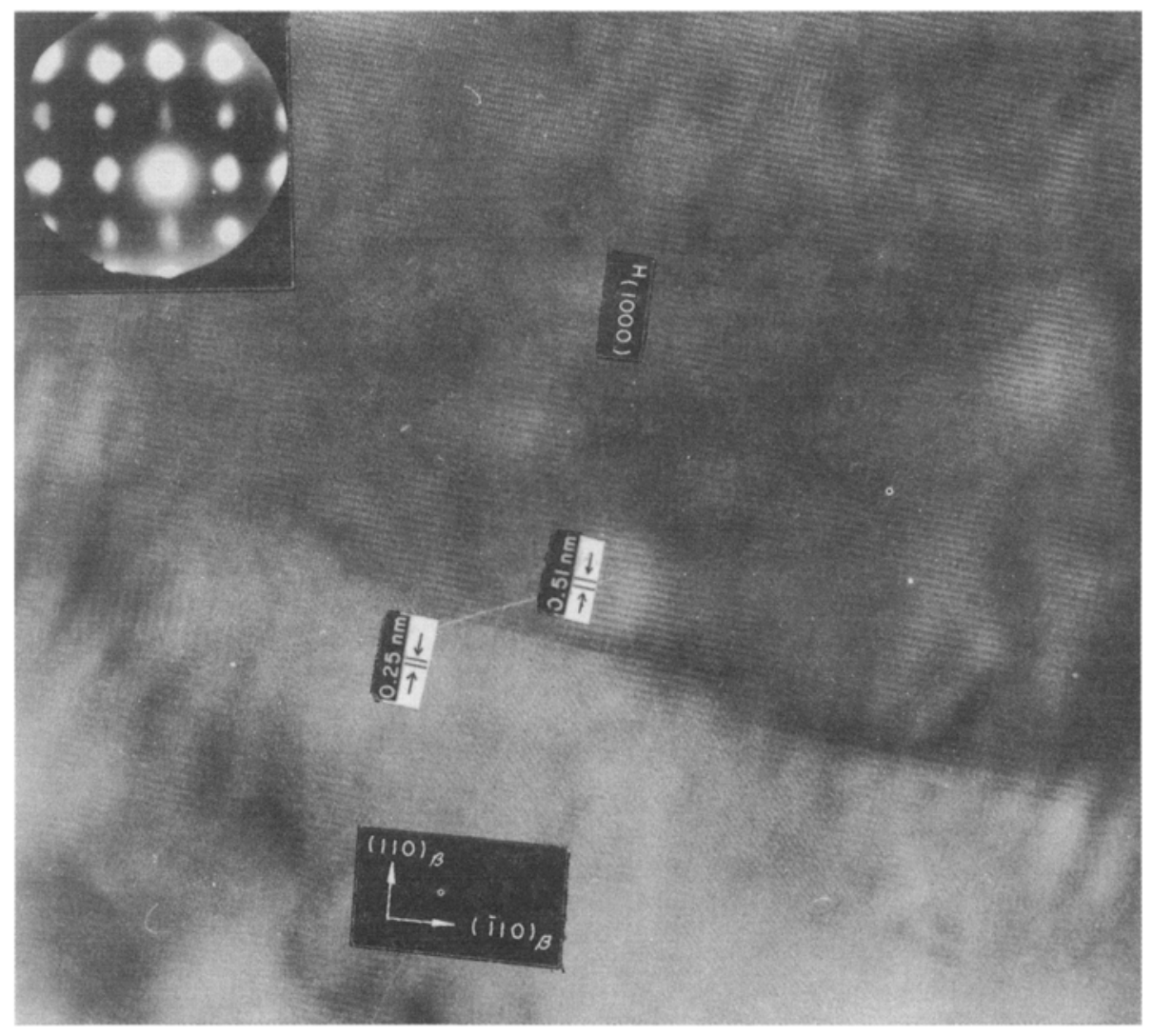

Figure 5. HREM image of a $\alpha / \beta$ interface in $\mathrm{Zr}-2.5 \mathrm{Nb}$ alloy. 
originates either due to the coupling of the APBs with the pre-existing boundaries or by the meeting of the two ordered domains leading to direct formation of a mixed boundary. It has been emphasized that the first demonstration of the MBs constitutes an important step in the direction of the understanding of the lamellar structure formation mechanism in two-phase TiAl formation. A description is given here of the HREM observations made on a TiAl alloy made by micropyretic synthesis. In this alloy the microstructure is lamellar with the lamellae being mostly of the $\gamma$ phase, the $\alpha_{2}$ phase occupying the in between space in some regions. Figure 4a shows this microstructure. It can be seen that though the microstructure comprises lamellae of the $\gamma$ and $\alpha_{2}$ phases, these lamellae are not alternately arranged. Instead the $\gamma$ lamellae share boundaries with another $\gamma$ lamellae in many instances. The HREM image of the microstructure is shown in figure $4 \mathrm{~b}$. It can be seen in this picture that the $\gamma / \gamma$ boundary is a twin boundary. The $\gamma-\alpha_{2}$ interface in this alloy was found to be similar to that obtained in the conventionally processed alloy at an atomic level. On this interface it was possible to see the formation of ledges. Various types of faults could be seen in lamellae of both the phases. In addition to the study of the interfaces, the structure imaging of the various phases occurring in the microstructures of these alloys has also been carried out and these will be reported elsewhere (Dey 1998).

\section{$4.4 \mathrm{Zr} 2.5 \mathrm{Nb}$ alloy}

This alloy finds application as pressure tubes in the CANDU type reactors. Several efforts have been made to understand the microstructure of this alloy and develop a microstructure, which has the best suitable properties. This alloy has a two-phase microstructure comprising the $\alpha$ and $\beta$ phases. A variety of interfaces have been encountered in this alloy out of which the important types are the $\alpha / \alpha$ and the $\alpha / \beta$ interface. A detailed examination of the $\alpha / \alpha$ interface has been carried out, with due emphasis on the dislocation arrangement at these interfaces and these observations have been reported by Srivastava et al (1995). The $\alpha / \beta$ interface has been examined by HREM in order to identify the atomic registry at these interfaces. HREM image of such an interface is shown in figure 5 of a specimen of $\mathrm{Zr}$ $2.5 \mathrm{Nb}$ in which dynamic recrystallization has occurred during hot extrusion. The HREM image indicated that the two lattices obeyed a Burger's orientation relationship. It also indicated that the planar matching between the $(0001)_{\alpha}$ and $(110)_{\beta}$ was near perfect ruling out the possibility of the existence of another type of orientation relationship known as the Potter orientation relationship (Srivastava et al 1995). It was possible to see three to five basal plane thick lattice registry ledges at these interfaces. The interfaces across which the lattices obey Burger's orientation relationship are known to provide a low energy configuration. Study of interfaces of this type by using lattice resolution establishes the exact nature of the orientation relationship, their state of energy and whether these can migrate easily leading to growth and decay of adjacent phases.

\section{Acknowledgements}

The authors wish to thank Dr P Mukhopadhyay, Dr M Sunderaraman, Dr U D Kulkarni, Dr R Ramanujam and Dr K Madangopal for many helpful discussions.

\section{References}

Banerjee S, Kulkarni U D and Urban K 1988 Acta Metall. 3735 Banerjee S and Madangopal K 1996 Metals Mater. \& Process 81966

Chandrasekhar M 1993 Metastable microstructures-Principles of design and applications (eds) D Banerjee and L A Jacobson (New Delhi: Oxford and IBH Publishing) p. 185

Dey G K 1998 unpublished work

Dey G K, Kulkarni U D, Batra I S and Banerjee S 1994 Acta Metall. Mater. 422973

Dey G K, Savalia R T, Baburaj E G and Banerjee S $1998 \mathrm{~J}$. Mater. Res. 13504

Goodhew P J and Humphreys F J 1987 Electron microscopy and analysis (London: Taylor and Francis) Second ed.

Hirabayashi M and Hiraga K 1987 Mater. Sci. Forum 2245

Kim Y W 1992 Acta Metall. Mater. 401121

Knowles K M 1982 Philos. Mag. A45 357

Kulkami U D and Banerjee S 1988 Acta Metall. 36413

Nishida M, Yamauchi K, Itai I, Ohgi H and Chiba A 1995 Acta Metall. Mater. 431229

Nissen H U and Beeli C 1991 in High resolution electron microscopy: Fundamentals and applications (eds) J Heydenreich and W Neumann (Halle/Saale, Germany: Institute fur Festkorperphysik und Electronmikroskopie) p. 272

Savalia R T, Baburaj E G, Dey G K and Banerjee S 1996 Acta Metall. Mater. 34625

Schryvers D and Tanner L E 1990 Mater. Sci. Forum 56329

Shindo D, Hirabayashi M, Kikuchi M, Kobayashi M and Syono Y 1988 Jpn. J. Appl. Phys. L27 1848

Singh S R and Howe J M 1992 Philos. Mag. A66 739

Spence J C H 1988 Experimental high resolution transmission electron microscopy (Amsterdam: North Holland)

Srivastava D, Dey G K and Banerjee S 1995 Met. and Mater. Trans. A26 2707

Tewari R, Banerjee S and Benderski L A 1998 Unpublished work Van Landuyt J, Van Tendeloo G and Amelinckx S 1991 in High resolution electron microscopy: Fundamentals and applications (eds) J Heydenreich and W Neumann (Halle/Saale, Germany: Institute fur Festkorperphysik und Electronmikroskopie) p. 254

Williams D B and Carter C B 1996 Transmission electron microscopy III (New York: Plenum Press)

Yang Y S and Wu S K 1992a Philos. Mag. A65 15

Yang Y S, Wu S K and Wang J W 1993 Philos. Mag. A67 463 